\title{
Relating relapse and $\mathrm{T} 2$ lesion changes to disability progression in multiple sclerosis: a systematic literature review and regression analysis
}

Kyle Fahrbach ${ }^{1 *}$, Rachel Huelin ${ }^{1}$, Amber L Martin ${ }^{1}$, Edward Kim² ${ }^{2}$ Homa B Dastani ${ }^{3}$, Stephen Rao ${ }^{4}$ and Manoj Malhotra ${ }^{5}$

\begin{abstract}
Background: In the treatment of multiple sclerosis (MS), the most important therapeutic aim of disease-modifying treatments (DMTs) is to prevent or postpone long-term disability. Given the typically slow progression observed in the majority of relapsing-remitting MS (RRMS) patients, the primary endpoint for most randomized clinical trials (RCTs) is a reduction in relapse rate. It is widely assumed that reducing relapse rate will slow disability progression. Similarly, MRI studies suggest that reducing T2 lesions will be associated with slowing long-term disability in MS. The objective of this study was to evaluate the relationship between treatment effects on relapse rates and active T2 lesions to differences in disease progression (as measured by the Expanded Disability Status Scale [EDSS]) in trials evaluating patients with clinically isolated syndrome (CIS), RRMS, and secondary progressive MS (SPMS).

Methods: A systematic literature review was conducted in Medline, Embase, CENTRAL, and PsycINFO to identify randomized trials published in English from January 1, 1993-June 3, 2013 evaluating DMTs in adult MS patients using keywords for CIS, RRMS, and SPMS combined with keywords for relapse and recurrence. Eligible studies were required to report outcomes of relapse and T2 lesion changes or disease progression in CIS, RRMS, or SPMS patients receiving DMTs and have a follow-up duration of at least 22 months. Ultimately, 40 studies satisfied these criteria for inclusion. Regression analyses were conducted on RCTs to relate differences between the effect of treatments on relapse rates and on active $\mathrm{T} 2$ lesions to differences between the effects of treatments on disease progression (as measured by EDSS).
\end{abstract}

Results: Regression analysis determined there is a substantive clinically and statistically significant association between concurrent treatment effects in relapse rate and EDSS; $p<0.01$. Lower treatment effects were associated with higher relative rates of disease progression. Significant associations between T2 lesion measures and EDSS measures also were found $(p<0.05)$, with some suggestion that the strength of the association may differ for older versus newer DMTs.

Conclusions: Treatment differences in relapse reduction and T2 lesions are positively related to differences in disease progression over the first two years of treatment.

Keywords: Relapsing-remitting multiple sclerosis, Secondary progressive multiple sclerosis, Relapse, Disability progression, $\mathrm{T} 2$ lesions

\footnotetext{
* Correspondence: Kyle.Fahrbach@evidera.com

'Evidera, 430 Bedford Street, Suite 300, Lexington, MA 02420, USA

Full list of author information is available at the end of the article
} 


\section{Background}

In the treatment of multiple sclerosis (MS), the most important therapeutic aim of disease-modifying treatments (DMTs) is to prevent or postpone long-term disability, typically defined by worsening on the Expanded Disability Status Scale (EDSS) [1]. Given the normally slow progression observed in the majority of relapsing-remitting MS patients (RRMS) [2], the primary endpoint for most randomized clinical trials (RCTs) is a reduction in relapse rate. It is widely assumed that reducing relapse rate will slow disability progression [3]. Similarly, magnetic resonance imaging (MRI) studies suggest that reducing T2 lesions [4], another short-term outcome in RCTs, will be associated with slowing long-term disability in MS [5].

Sormani and colleagues performed a quantitative metaanalysis of the predictive power of annualized relapse rates and new/enlarged T2 lesions on EDSS progression across a wide range of RCTs for RRMS [6]. Their results indicated that the therapeutic benefit of the drugs, defined by relapse rates and T2 lesions, generally correlated with disability progression. The Sormani et al. study, however, included an intercept in their regression analyses. Inclusion of a non-zero intercept implies that two treatments that do not differ in affecting the predictor (i.e., two treatments that have the same relapse rate) will differ in affecting the outcome (i.e., they will lead to different amounts of EDSS progression). While it is conceptually possible for some pairs of treatments, it would have to hold for all pairs of treatments for the regression to be generalizable. Furthermore, their analysis was limited to RCTs directed at RRMS patients, thus restricting the conclusions that can be drawn regarding the predictive relationship between relapse rates and disability progression in other forms of MS.

In this quantitative meta-analysis, we have extended the Sormani et al. analyses by including data from DMTs involving secondary progressive MS (SPMS) patients. In addition, we have expanded the MRI predictors to include T2 lesion volume, a potentially better predictor of disability progression than new and enlarging $\mathrm{T} 2$ lesions. Finally, our statistical approach, which excludes the use of an intercept in the regression analyses, should provide a more meaningful prediction of the relative risk of EDSS progression from treatment differences in the surrogate endpoints of interest (relapse rate and T2 lesions).

\section{Methods}

To identify and retrieve all potentially relevant trials assessing treatment to delay or avoid relapse and disability progression in patients with RRMS, SPMS, and clinically isolated syndrome (CIS), we conducted literature searches in Medline (via PubMed), Embase, Cochrane Central Register of Clinical Trials (CENTRAL), and PsycINFO. The following algorithm was used in PubMed and analogous searches were developed for the remaining databases:
1. "Multiple Sclerosis, Relapsing-Remitting"[Mesh] OR "Relapsing-Remitting Multiple Sclerosis" OR "secondary progressive multiple sclerosis" OR ("Multiple Sclerosis"[Mesh] AND "secondary progressive" [TIAB]) OR "clinically isolated syndrome" OR "early MS" OR "early multiple sclerosis" OR "clinically definite MS" OR "clinically definite multiple sclerosis".

2. relapse OR relapses OR relapsed OR recurrence.

3. \#1 AND \#2.

Limits: Humans, English, clinical trial, Not reviews, editorials, comments or case reports, and published between January 1, 1993 and June 3, 2013, with an abstract.

Eligible studies included RCTs of at least 22 months' duration assessing treatment of MS with DMTs that reported both relapse and disability progression. The Cochrane Library was also searched for recent systematic reviews of the subject, which could be used as a source of further references. A manual check of reference lists from all included studies and relevant reviews/ meta-analyses was performed to supplement the above searches and ensure a comprehensive review. Conference abstracts and unpublished literature were not included.

\section{Study selection}

The full text articles of accepted abstracts that passed the initial screening underwent review by investigators trained in systematic review procedures and each excluded study required the consensus of an independent investigator. Abstracts were included when all of the following were true: the study was an RCT evaluating a minimum of 20 adults with CIS, RRMS, or SPMS with at least 22 months of follow-up in which relapse rate or MRI lesion data and disability progression related to treatment with DMTs (both approved and non-approved) were reported, and the study was published within the search period (January 1, 1993-June 3, 2013) for this review.

\section{Data extraction}

Data were collected into an electronic database developed specifically for this review by a single investigator and independently verified by a second investigator. Discrepancies in data extraction were reviewed by the two investigators, and when necessary, any unresolved discrepancies were resolved by a third investigator. The endpoints sought for data capture included annualized relapse rate (ARR), mean change in EDSS, the proportion of patients with disability progression, as well as counts and volume changes for T2 and gadolinium lesions. Definitions for relapse and disease progression were also extracted as defined by authors to ensure similar methods were used in determining the presence of these endpoints across studies. 


\section{Statistical methods}

Regression analyses were conducted on RCTs to relate differences between the effect of treatments on relapse rates and on active T2 lesions, to differences between the effects of treatments on disability progression, as measured by EDSS.

The statistical methods used were similar to methods outlined in meta-analyses conducted by Johnson et al. [7] and Sormani et al. [6]. Specifically, weighted leastsquares regressions (in which the weight is the total sample size of the two arms being compared) were conducted for combinations of predictor and outcome listed below. However, the analyses were not identical; unlike Sormani et al. [6], we did not weight by duration of follow-up and did not include an intercept in the regression analyses. As noted in the introduction, inclusion of a non-zero intercept implies that two treatments with similar relapse rates could lead to a different degree of EDSS progression. While this is conceptually possible for some pairs of treatments, it would have to hold for all pairs of treatments for the regression to be generalizable.

Regressions were conducted for the following combinations of strata and predictor/outcome pairings:

\section{Stratifications}

- RRMS, limited to studies in which both arms have approved DMTs (e.g., excluding studies with alemtuzumab, statin-add-on, azathioprine-add-on, cladribine tablets, MBP8298).

- All RRMS, all DMTs.

- SPMS/SPMS mixed.

Overall analyses across all disease courses (including CIS) were also planned; however, given the important clinical differences between disease stages with regard to baseline rates of relapse, it was judged more appropriate to analyze RRMS and SPMS studies independently. CIS studies were not investigated independently, given the sparseness of data available.

Regressions were conducted for the following combinations of strata and predictor/outcome pairings:

\section{Predictors/outcomes}

- Ratio of ARR as a predictor of the relative risk of EDSS progression (i.e., the ratio of the proportions of patients with a predefined threshold of EDSS increase).

- Ratio in number of new (or new/enlarged) T2 lesions as a predictor of the relative risk of EDSS progression.

- Ratio of follow-up median T2 lesion volumes as a predictor of the relative risk of EDSS progression.
One other analysis was planned a priori; however, it was not feasible due to sparseness of data:

- Ratio of gadolinium (GD) lesion volume as a predictor of the relative risk of EDSS progression (i.e., the ratio of the proportions of patients with a predefined threshold of EDSS increase).

In cases where the relative risk of EDSS progression was not available or calculable, the hazard ratio for timeto-progression was used if it was available.

All calculations were performed using $\mathrm{SAS}^{\oplus}$ software version 9.2 and SPSS $^{\oplus}$ software version 15.0.

\section{Results}

A total of 1,104 unique citations were identified for review at the abstract level. Of these, 91 abstracts were selected for further review as full-text articles, and 1,013 were excluded. The primary reason for exclusion following full text review was study duration less than 22 months $(n=23)$, followed by no extractable outcomes of interest $(n=20)$. Figure 1 presents the study attrition of the literature review. Forty primary articles [8-47] that examined relapse rate and disability progression related to treatment with DMTs in adults with CIS, RRMS, or SPMS with at least 22 months of follow-up were identified for inclusion after full text review.

Of the 40 identified studies, the majority were on RRMS populations (30), followed by SPMS (7). There were only two CIS studies that met our inclusion criteria, as well as one trial evaluating a mixed RRMS and SPMS population.

In the figures representing the analyses (Figures 2, 3 and 4), each slope suggests a predicted difference between any two treatments on an outcome (e.g., log-relative risk of EDSS progression) given a difference between those same treatments on a predictor (e.g., log-rate ratio of the ARRs). The trial data contributing to the analyses are provided in Tables 1 and 2. Evidence tables show the data for the predictors (i.e., ARR ratio, final T2 lesion volume ratio, ratio in number of new/enlarged T2 lesions) and the outcome (ratio of patients with confirmed disease progression).

\section{Predicting disability progression from relapse}

The relationships between the log-ARR and log-relativerisk of EDSS progression were statistically significant across all analyses $(p<0.001$ for RRMS-all DMTs and RRMSapproved DMTs, $p=0.027$ for SPMS; see Table 3). The strength of the predictive power of log-ARR to predict EDSS progression did not vary substantively across analyses (slopes of $0.53,0.50$, and 0.40 , respectively). Figure 2 plots the relationship between the log-ARR and logrelative risk of EDSS progression for all RRMS studies. 
1,104 titles and abstracts identified from literature

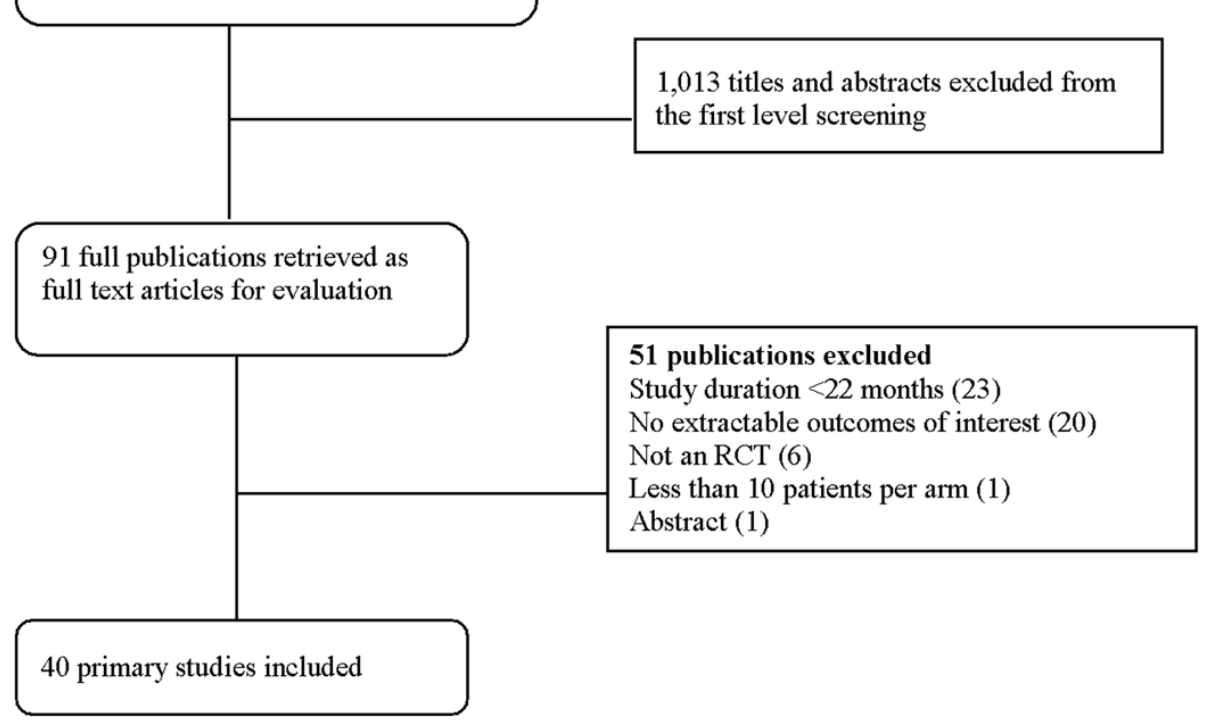

Figure 1 Study attrition.

Each point in the plot represents a study comparison for these two effects. For instance, the most rightward point is from Gonsette et al. [23], which found almost no difference in EDSS progression between the two arms (approximately $18 \%$ for both) but a substantive difference in relapse rate $(0.50$ vs. 0.38 , for a log-rate-ratio of 0.27). Each study with two arms (one treatment comparison, e.g., treatment A vs. treatment B) with sufficient data contributed one data point to the analysis; studies with three arms (two treatment comparisons, e.g., A vs. $\mathrm{B}$ and $\mathrm{A}$ vs. C) contributed two data points.

Any given slope can be interpreted by determining what difference between treatments in EDSS progression one would expect given a realistic difference in ARR ratio. A realistic difference in the ARR ratio can be operationalized as the median ARRR across all studies. In this data, the median ratio of the mean relapse rate in the active to the control group was 0.70. For instance, in

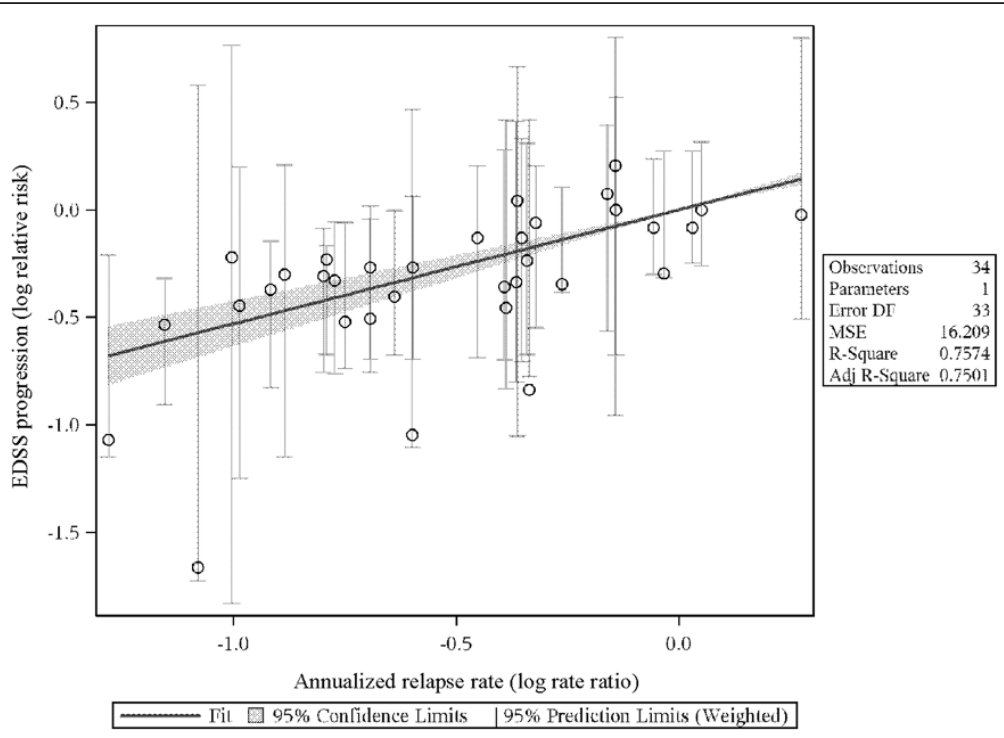

Figure 2 Predicting log-relative risk of EDSS from log-rate ratio of relapse rate: RRMS, all DMTs. EDSS: Expanded Disability Status Scale; Log RR. 


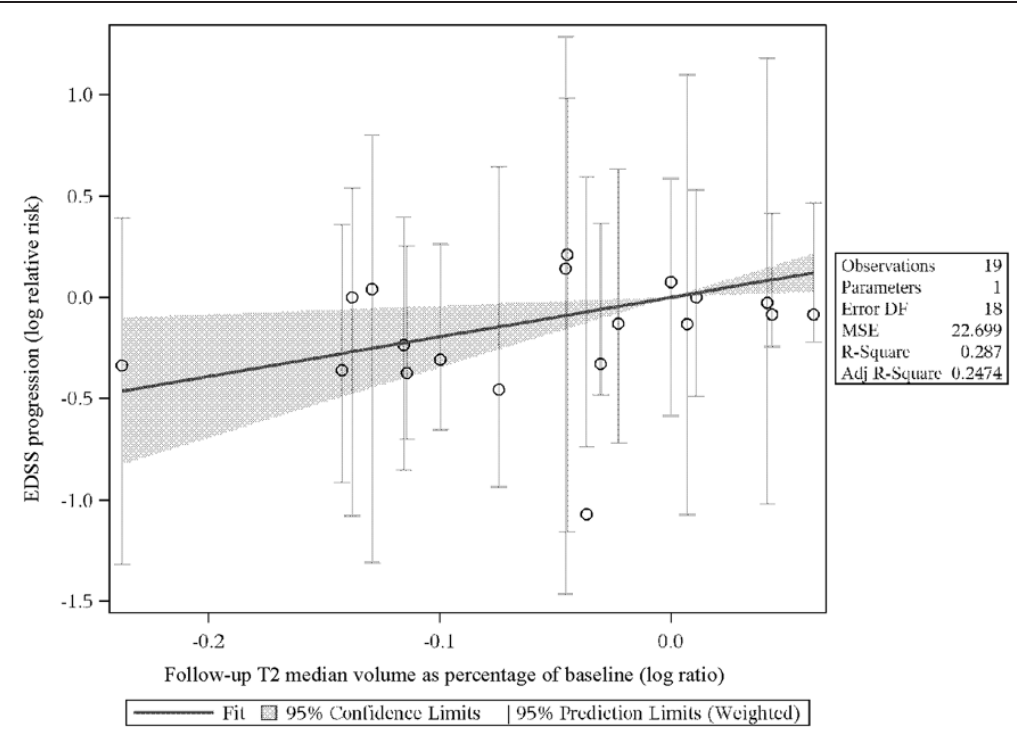

Figure 3 Predicting log-relative risk of EDSS from log-rate ratio of median T2 lesion volume: RRMS, all DMTs. EDSS: Expanded Disability Status Scale.

Johnson et al. [27], the ARR for glatiramer acetate (GA) was 0.59 , while it was 0.84 for placebo (relative risk $[R R]=0.70)$.

As $\ln (0.70)=-0.353$, the predicted log-relative-risk of EDSS progression in studies like Johnson et al. is:

$$
\ln \left(\mathrm{RR}_{\mathrm{EDSS}}\right)=\text { Slope } * \ln (\mathrm{ARRR})
$$

Or

$$
\ln \left(\operatorname{RR}_{\mathrm{EDSS}}\right)=0.53(-0.353),=-0.187 \text {. }
$$

As $\exp (-0.187)=0.83$, we can predict that the relative risk of EDSS progression in studies like Johnson et al. (i.e. with an ARRR of 0.70 ) will be 0.83. In Johnson et al., the relative risk of EDSS progression was similar to this value: it was $0.88(21.6 \%$ progression in GA, $24.6 \%$ progression on placebo).

\section{Predicting disability progression from lesion volume}

The slope for predicting log-relative-risk of EDSS progression from the log-ratio of median follow-up in T2 volume (expressed as a percentage of baseline volume) was statistically significant for the RRMS subset of studies $(1.95,95 \%$ CI $[0.43,3.48], p=0.015)$ and marginally statistically significant for the subset of RRMS studies on approved DMTs $(1.09,95 \%$ CI $[-0.09,2.27], p=0.067)$.

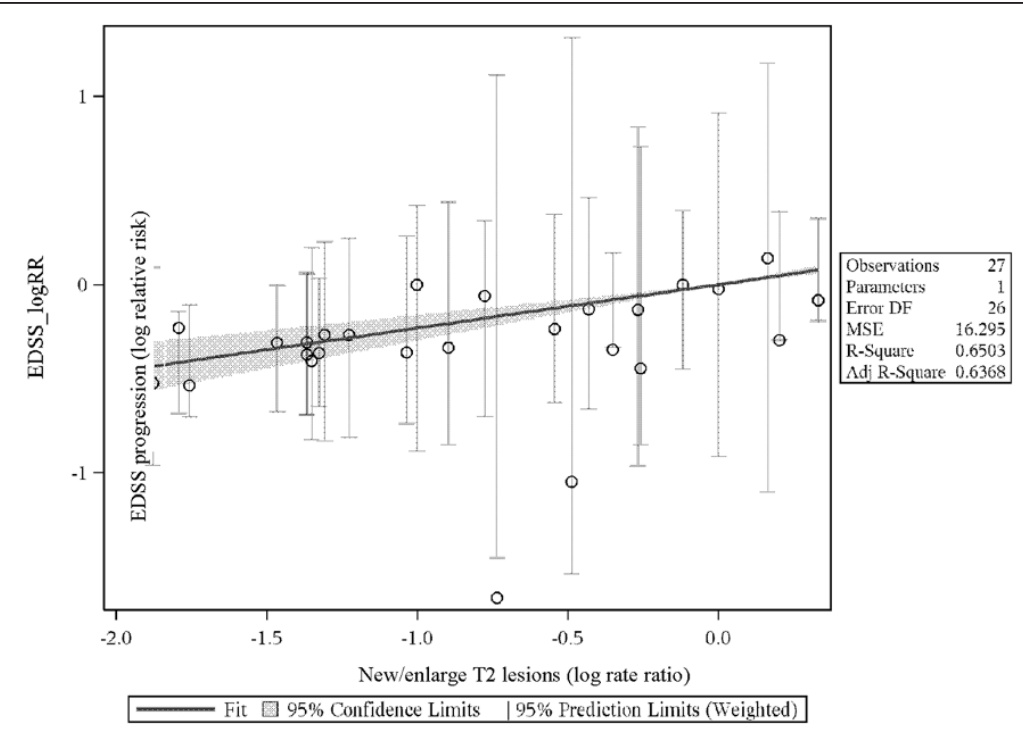

Figure 4 Predicting log-relative risk of EDSS from log-rate ratio of active T2 lesions count. EDSS: Expanded Disability Status Scale. 
Table 1 Study summaries in trials reporting relapse and disability progression

\begin{tabular}{|c|c|c|c|c|c|c|c|c|c|}
\hline Trial & $\begin{array}{l}\text { Follow-up } \\
\text { (months) }\end{array}$ & Comparison & $\begin{array}{l}\text { Patients } \\
\text { analyzed }\end{array}$ & $\begin{array}{c}\text { ARR } \\
\text { experimental } \\
\text { arm } \\
\end{array}$ & $\begin{array}{c}\text { ARR } \\
\text { control arm }\end{array}$ & $\begin{array}{l}\text { Relapse rate } \\
\text { ratio }\end{array}$ & $\begin{array}{c}\text { EDSS } \\
\text { progression } \\
\text { experimental arm }\end{array}$ & $\begin{array}{c}\text { EDSS } \\
\text { Progression } \\
\text { control arm } \\
\end{array}$ & $\begin{array}{l}\text { Disability } \\
\text { risk ratio }\end{array}$ \\
\hline $\begin{array}{l}\text { Comi G, } \\
\text { et al., [17] }\end{array}$ & 24 & $\begin{array}{l}\text { IFNB-1a } 22 \mu \mathrm{g} \text { vs. } \\
\text { Placebo }\end{array}$ & 309 & 0.33 & 0.43 & 0.767 & 15.00 & 20.00 & 0.750 \\
\hline $\begin{array}{l}\text { Hartung } \\
\text { HP, et al. } \\
{[24]}\end{array}$ & 36 & $\begin{array}{l}\text { Mitoxantrone } 12 \mathrm{mg} \\
\text { vs. Placebo }\end{array}$ & 124 & 0.35 & 1.02 & 0.343 & 8.00 & 22.00 & 0.364 \\
\hline $\begin{array}{l}\text { Durelli } L \text {, } \\
\text { et al. [18] }\end{array}$ & 24 & $\begin{array}{c}\text { IFNB-1a } 30 \mu \mathrm{g} \text { vs. IFNB- } \\
\text { 1b } 250 \mu \mathrm{g}\end{array}$ & 188 & 0.50 & 0.70 & 0.714 & 13.00 & 30.00 & 0.433 \\
\hline $\begin{array}{l}\text { Rudick RA, } \\
\text { et al. [38] }\end{array}$ & 24 & $\begin{array}{c}\text { IFNB-1a plus } \\
\text { Natalizumab vs. IFNB- } \\
1 \mathrm{a}\end{array}$ & 1,171 & 0.34 & 0.75 & 0.453 & 23.00 & 29.00 & 0.793 \\
\hline $\begin{array}{l}\text { Havrdova E, } \\
\text { et al. [25] }\end{array}$ & 24 & $\begin{array}{c}\text { IFNB-1a plus } \\
\text { azathioprinethioprine } \\
\text { vs. IFNB-1a }\end{array}$ & 116 & 0.91 & 1.05 & 0.867 & 20.70 & 16.80 & 1.232 \\
\hline $\begin{array}{l}\text { Havrdova E, } \\
\text { et al. [25] }\end{array}$ & 24 & $\begin{array}{c}\text { IFNB-1a plus } \\
\text { azathioprine plus } \\
\text { prednisone vs. IFNB-1a }\end{array}$ & 120 & 0.73 & 1.05 & 0.695 & 17.50 & 16.80 & 1.042 \\
\hline $\begin{array}{l}\text { Mikol DD, } \\
\text { et al. [32] }\end{array}$ & 22 & $\begin{array}{l}\text { IFNB-1a vs. Glatiramer } \\
\text { acetate }\end{array}$ & 764 & 0.29 & 0.30 & 0.967 & 8.70 & 11.70 & 0.744 \\
\hline $\begin{array}{l}\text { Ravnborg } \\
\text { M, et al. } \\
{[37]}\end{array}$ & 36 & $\begin{array}{c}\text { IFNB-1a plus } \\
\text { Methylprednisolone vs. } \\
\text { IFNB-1a }\end{array}$ & 338 & 0.21 & 0.33 & 0.636 & 25.00 & 28.50 & 0.877 \\
\hline $\begin{array}{l}\text { Sorensen P, } \\
\text { et al. [39] }\end{array}$ & 22 & $\begin{array}{c}\text { IFNB-1a plus } \\
\text { Methylprednisolone vs. } \\
\text { IFNB-1a }\end{array}$ & 130 & 0.22 & 0.59 & 0.373 & 16.00 & 25.00 & 0.640 \\
\hline $\begin{array}{l}\text { O'Connor } \\
\text { P, et al. [34] }\end{array}$ & 42 & $\begin{array}{l}\text { Glatiramer acetate vs. } \\
\text { IFNB-1b } 250 \mu \mathrm{g}\end{array}$ & 1,158 & 0.34 & 0.36 & 0.944 & 20.50 & 22.30 & 0.919 \\
\hline $\begin{array}{l}\text { O'Connor } \\
\text { P, et al. [34] }\end{array}$ & 42 & $\begin{array}{l}\text { Glatiramer acetate vs. } \\
\text { IFNB-1b } 500 \mu \mathrm{g}\end{array}$ & 1,100 & 0.34 & 0.33 & 1.030 & 20.50 & 22.30 & 0.919 \\
\hline $\begin{array}{l}\text { Gonsette } \\
\text { RE, et al. } \\
\text { [23] }\end{array}$ & 24 & $\begin{array}{l}\text { IFNB-1b plus Inosine } \\
\text { vs. IFNB-1b }\end{array}$ & 157 & 0.50 & 0.38 & 1.316 & 17.78 & 18.18 & 0.978 \\
\hline $\begin{array}{l}\text { Coles AJ, } \\
\text { et al. [16] }\end{array}$ & 36 & $\begin{array}{l}\text { Alemtuzumab vs. IFNB- } \\
1 \mathrm{a}\end{array}$ & 333 & 0.1 & 0.36 & 0.278 & 9.00 & 26.20 & 0.344 \\
\hline $\begin{array}{l}\text { Kappos } L, \\
\text { et al. [30] }\end{array}$ & 24 & $\begin{array}{l}\text { Fingolimod } 0.5 \text { mg vs. } \\
\text { Placebo }\end{array}$ & 843 & 0.18 & 0.40 & 0.450 & 17.70 & 24.10 & 0.734 \\
\hline $\begin{array}{l}\text { Kappos } L, \\
\text { et al. [30] }\end{array}$ & 24 & $\begin{array}{c}\text { Fingolimod } 1.25 \text { mg vs. } \\
\text { Placebo }\end{array}$ & 847 & 0.16 & 0.40 & 0.400 & 16.60 & 24.10 & 0.689 \\
\hline $\begin{array}{l}\text { Polman CH, } \\
\text { et al. [36] }\end{array}$ & 24 & $\begin{array}{l}\text { Natalizumab vs. } \\
\text { Placebo }\end{array}$ & 942 & 0.23 & 0.73 & 0.315 & 17.00 & 29.00 & 0.586 \\
\hline $\begin{array}{l}\text { Clanet M, } \\
\text { et al. [14] }\end{array}$ & 36 & $\begin{array}{c}\text { IFNB-1a } 30 \mu \mathrm{g} \text { vs. IFNB- } \\
\text { 1a } 60 \mu \mathrm{g}\end{array}$ & 802 & 0.81 & 0.77 & 1.052 & 37.00 & 37.00 & 1.000 \\
\hline $\begin{array}{l}\text { Ebers GC, } \\
\text { et al. [9] }\end{array}$ & 104 & $\begin{array}{c}\text { IFNB-1a } 22 \mu \mathrm{g} \text { vs. } \\
\text { Placebo }\end{array}$ & 376 & 0.91 & 1.28 & 0.711 & 30.00 & 38.00 & 0.789 \\
\hline $\begin{array}{l}\text { Ebers GC, } \\
\text { et al. [9] }\end{array}$ & 104 & $\begin{array}{l}\text { IFNB-1a } 44 \mu \mathrm{g} \text { vs. } \\
\quad \text { Placebo }\end{array}$ & 373 & 0.87 & 1.28 & 0.676 & 26.50 & 38.00 & 0.697 \\
\hline $\begin{array}{l}\text { Johnson } \\
\text { KP, et al. } \\
\text { [27] }\end{array}$ & 24 & $\begin{array}{c}\text { Glatiramer acetate vs. } \\
\text { Placebo }\end{array}$ & 251 & 0.59 & 0.84 & 0.702 & 21.60 & 24.60 & 0.878 \\
\hline $\begin{array}{l}\text { Achiron A, } \\
\text { et al. [11] }\end{array}$ & 24 & $\begin{array}{l}\text { Immunoglobulin vs. } \\
\text { Placebo }\end{array}$ & 40 & 0.59 & 1.61 & 0.366 & 13.70 & 17.10 & 0.801 \\
\hline $\begin{array}{l}\text { Baumhackl } \\
\text { U, et al. } \\
\text { [13] }\end{array}$ & 24 & $\begin{array}{c}\text { Hydrolytic enzymes vs. } \\
\text { Placebo }\end{array}$ & 291 & 0.63 & 0.74 & 0.851 & 28.00 & 26.00 & 1.077 \\
\hline $\begin{array}{l}\text { Fazekas F, } \\
\text { et al. [20] }\end{array}$ & 24 & $\begin{array}{l}\text { Immunoglobulin vs. } \\
\text { Placebo }\end{array}$ & 148 & 0.52 & 1.26 & 0.413 & 17.00 & 23.00 & 0.739 \\
\hline
\end{tabular}


Table 1 Study summaries in trials reporting relapse and disability progression (Continued)

\begin{tabular}{|c|c|c|c|c|c|c|c|c|c|}
\hline $\begin{array}{l}\text { Millefiorini } \\
\text { E, et al.[33] }\end{array}$ & 24 & $\begin{array}{l}\text { Mitoxantrone vs. } \\
\text { Placebo }\end{array}$ & 51 & 0.445 & 1.31 & 0.340 & 7.00 & 37.00 & 0.189 \\
\hline $\begin{array}{l}\text { Jacobs LD, } \\
\text { et al. [26] }\end{array}$ & 24 & IFNB-1a vs. Placebo & 172 & 0.61 & 0.90 & 0.678 & 21.10 & 33.30 & 0.633 \\
\hline $\begin{array}{l}\text { Ebers GC, } \\
\text { et al. [8] }\end{array}$ & 36 & $\begin{array}{c}\text { IFNB-1b 1.6 MIU vs. } \\
\text { Placebo }\end{array}$ & 228 & 1.05 & 1.21 & 0.868 & 28.00 & 28.00 & 1.000 \\
\hline $\begin{array}{l}\text { Ebers GC, } \\
\text { et al. [8] }\end{array}$ & 36 & $\begin{array}{l}\text { IFNB-1b } 8 \text { MIU vs. } \\
\text { Placebo }\end{array}$ & 227 & 0.84 & 1.21 & 0.694 & 20.00 & 28.00 & 0.714 \\
\hline $\begin{array}{l}\text { van de } \\
\text { Wyngaert } \\
\text { FA, et al. } \\
{[40]}\end{array}$ & 36 & $\begin{array}{l}\text { Mitoxantrone vs. } \\
\text { Methylprednisolone }\end{array}$ & 28 & 0.26 & 1.00 & 0.260 & 21.00 & 21.00 & 1.000 \\
\hline $\begin{array}{l}\text { Andersen } \\
\text { O et al. [12] }\end{array}$ & 36 & $\begin{array}{l}\text { IFNB-1a } 22 \mu \mathrm{g} \text { vs. } \\
\text { Placebo }\end{array}$ & 364 & 0.25 & 0.27 & 0.926 & 41.00 & 38.00 & 1.079 \\
\hline $\begin{array}{l}\text { Cohen JA, } \\
\text { et al. [15] }\end{array}$ & 24 & $\begin{array}{c}\text { IFNB-1a } 60 \mu \mathrm{g} \text { vs. } \\
\text { Placebo }\end{array}$ & 379 & 0.20 & 0.30 & 0.667 & 28.50 & 33.70 & 0.846 \\
\hline $\begin{array}{l}\text { Panitch H, } \\
\text { et al. [35] }\end{array}$ & 36 & $\begin{array}{l}\text { IFNB-1b } 160 \mu \mathrm{\mu g} \text { vs. } \\
\text { Placebo }\end{array}$ & 622 & 0.20 & 0.28 & 0.714 & 39.00 & 34.00 & 1.147 \\
\hline $\begin{array}{l}\text { Panitch H, } \\
\text { et al. [35] }\end{array}$ & 36 & $\begin{array}{l}\text { IFNB-1b } 250 \mu \mathrm{g} \text { vs. } \\
\quad \text { Placebo }\end{array}$ & 625 & 0.16 & 0.28 & 0.571 & 32.00 & 34.00 & 0.941 \\
\hline $\begin{array}{l}\text { Kappos L, } \\
\text { et al. [28] }\end{array}$ & 36 & IFNB-1b vs. Placebo & 718 & 0.44 & 0.64 & 0.688 & 38.90 & 49.70 & 0.783 \\
\hline $\begin{array}{l}\text { Edan G, } \\
\text { et al. [41] }\end{array}$ & 36 & $\begin{array}{l}\text { Mitoxantrone } 12 \mathrm{mg} / \\
\mathrm{m}^{2} \text { vs. IFNß-1b } 250 \mathrm{\mu g}\end{array}$ & 109 & 0.39 & 0.71 & 0.549 & 9.10 & 25.90 & 0.351 \\
\hline $\begin{array}{l}\text { Freedman } \\
\text { MS, et al. } \\
{[42]}\end{array}$ & 24 & $\begin{array}{l}\text { MBP8298 vs. Placebo in } \\
\text { DR2+ and/or DR4+ } \\
\text { haplotypes }\end{array}$ & 513 & 0.13 & 0.14 & 0.929 & 30.70 & 27.80 & 1.104 \\
\hline $\begin{array}{l}\text { Freedman } \\
\text { MS, et al. } \\
{[42]}\end{array}$ & 24 & $\begin{array}{l}\text { MBP8298 vs. Placebo in } \\
\text { DR2- and/or DR4- } \\
\text { haplotypes }\end{array}$ & 99 & 0.08 & 0.20 & 0.400 & 28.30 & 35.80 & 0.791 \\
\hline $\begin{array}{l}\text { Comi G, } \\
\text { et al. [45] }\end{array}$ & 24 & $\begin{array}{l}\text { Laquinimod } 0.6 \text { mg vs. } \\
\text { Placebo }\end{array}$ & 1,106 & 0.30 & 0.39 & 0.769 & 11.10 & 15.70 & 0.707 \\
\hline $\begin{array}{l}\text { Cohen JA, } \\
\text { et al. [43] }\end{array}$ & 24 & $\begin{array}{c}\text { Alemtuzumab } 12 \mathrm{mg} \\
\text { vs. IFN } 3-1 \mathrm{a} 44 \mathrm{\mu g}\end{array}$ & 563 & 0.18 & 0.39 & 0.462 & 8.00 & 11.12 & 0.719 \\
\hline $\begin{array}{l}\text { Coles AJ, } \\
\text { et al. [44] }\end{array}$ & 24 & $\begin{array}{l}\text { Alemtuzumab } 12 \mathrm{mg} \\
\text { vs. IFN } \beta-1 \mathrm{a} 44 \mathrm{\mu g}\end{array}$ & 667 & 0.26 & 0.52 & 0.500 & 12.71 & 21.13 & 0.602 \\
\hline $\begin{array}{l}\text { Fox RJ, } \\
\text { et al. [46] }\end{array}$ & 24 & $\begin{array}{l}\text { Dimethyl fumarate } \\
240 \text { mg BID vs. } \\
\text { Placebo }\end{array}$ & 480 & 0.22 & 0.40 & 0.550 & 13.00 & 17.00 & 0.765 \\
\hline $\begin{array}{l}\text { Fox RJ, } \\
\text { et al. [46] }\end{array}$ & 24 & $\begin{array}{l}\text { Dimethyl fumarate } \\
240 \text { mg TID vs. Placebo }\end{array}$ & 466 & 0.20 & 0.40 & 0.500 & 13.00 & 17.00 & 0.765 \\
\hline $\begin{array}{l}\text { Fox RJ, } \\
\text { et al. [46] }\end{array}$ & 24 & $\begin{array}{l}\text { Glatiramer acetate vs. } \\
\text { Placebo }\end{array}$ & 471 & 0.29 & 0.40 & 0.725 & 16.00 & 17.00 & 0.941 \\
\hline
\end{tabular}

ARR, annualized relapse rate; BID, twice daily; EDSS, Expanded Disability Status Scale; IFNB, Interferon-beta; $\mu \mathrm{g}$, microgram; mg, milligram; mg/m2, milligrams per square meter of body surface; MIU, million international units; TID, three times daily.

There were insufficient data on $\mathrm{T} 2$ volume to conduct analyses on SPMS patients; only one study [35] had such data. Figure 3 plots the relationship between the log-rate ratio of median T2 lesion volume and log-relative risk of EDSS progression for all RRMS studies.

Following the example above, the median ratio of follow-up T2 lesion volume was 0.96. For instance, in Coles et al. 2008,[16] the median T2 lesion volume dropped by $16.4 \%$ on alemtuzumab and dropped $13.3 \%$ on placebo. This led to a ratio of 0.96 for the follow-up values $(0.836 / 0.867)$ and a $\log$-ratio of -0.036 .
The predicted difference between treatments in EDSS progression in studies like Coles 2008 [16] is:

$$
\ln \left(\mathrm{RR}_{\mathrm{EDSS}}\right)=\text { Slope } * \ln (\text { ratio of median } \mathrm{T} 2 \text { volume }),
$$

Or

$$
\ln \left(\mathrm{RR}_{\mathrm{EDSS}}\right)=1.95 *(-0.036)=-0.070
$$

As $\exp (-0.070)=0.93$, we predict that the relative risk of EDSS progression in studies like Coles et al. 2008 will be 0.93 . The actual ratio in Coles et al. 2008 was much 
Table 2 Study summaries in trials reporting MRI changes and disability progression

\begin{tabular}{|c|c|c|c|c|c|c|c|c|c|c|c|c|}
\hline Trial & $\begin{array}{l}\text { Follow-up } \\
\text { (months) }\end{array}$ & Comparison & $\begin{array}{l}\text { Patients } \\
\text { Analyzed }\end{array}$ & $\begin{array}{c}\text { Change in } \mathrm{T} 2 \\
\text { Lesion Volume } \\
\text { Experimental } \\
\text { Arm }\end{array}$ & $\begin{array}{l}\text { Change in T2 } \\
\text { Lesion } \\
\text { Volume } \\
\text { Control Arm }\end{array}$ & $\begin{array}{l}\text { Final T2 } \\
\text { Lesion } \\
\text { Volume } \\
\text { Ratio }\end{array}$ & $\begin{array}{l}\text { New and/or } \\
\text { Enlarged T2 } \\
\text { Lesion } \\
\text { Experimental } \\
\text { Arm }\end{array}$ & $\begin{array}{c}\text { New and or } \\
\text { Enlarged T2 } \\
\text { Lesion Control } \\
\text { Arm }\end{array}$ & $\begin{array}{l}\text { T2 } \\
\text { Lesion } \\
\text { Count } \\
\text { Ratio }\end{array}$ & $\begin{array}{c}\text { EDSS } \\
\text { Progression } \\
\text { Experimental } \\
\text { Arm }\end{array}$ & $\begin{array}{c}\text { EDSS } \\
\text { Progression } \\
\text { Control Arm }\end{array}$ & $\begin{array}{l}\text { Disability } \\
\text { Risk Ratio }\end{array}$ \\
\hline $\begin{array}{l}\text { Kappos L, } \\
\text { et al. [29] }\end{array}$ & 24 & $\begin{array}{c}\text { IFNB-1b } 250 \mu \mathrm{g} \text { vs. } \\
\text { Placebo }\end{array}$ & 468 & -10.60 & -5.00 & 0.941 & 3.70 & 8.50 & 0.435 & 12.00 & 20.00 & 0.600 \\
\hline $\begin{array}{l}\text { Comi G, } \\
\text { et al. [17] }\end{array}$ & 24 & $\begin{array}{c}\text { IFNB-1a } 22 \mu \mathrm{g} \text { vs. } \\
\text { Placebo }\end{array}$ & 300 & -13.00 & 8.80 & 0.800 & 2.00 & 3.00 & 0.667 & 15.00 & 20.00 & 0.750 \\
\hline $\begin{array}{l}\text { Hartung HP, } \\
\text { et al. [24] }\end{array}$ & 36 & $\begin{array}{l}\text { Mitoxantrone } 12 \mathrm{mg} \\
\text { vs. Placebo }\end{array}$ & 124 & & & & 0.29 & 1.94 & 0.149 & 8.00 & 22.00 & 0.364 \\
\hline $\begin{array}{l}\text { Rudick RA, } \\
\text { et al. [38] }\end{array}$ & 24 & $\begin{array}{c}\text { IFNB-1a plus } \\
\text { Natalizumab vs. IFNB } \\
1 \mathrm{la}\end{array}$ & 1171 & & & & 0.90 & 5.40 & 0.167 & 23.00 & 29.00 & 0.793 \\
\hline $\begin{array}{l}\text { Havrdova E, } \\
\text { et al. [25] }\end{array}$ & 24 & $\begin{array}{c}\text { IFNB-1a plus } \\
\text { Azathioprinethioprine } \\
\text { vs. IFNB-1a }\end{array}$ & 88 & 24.60 & 30.30 & 0.956 & & & & 20.70 & 16.80 & 1.232 \\
\hline $\begin{array}{l}\text { Havrdova E, } \\
\text { et al. [25] }\end{array}$ & 24 & $\begin{array}{c}\text { IFNB-1a plus } \\
\text { azathioprine plus } \\
\text { prednisone vs. IFNB- } \\
\text { la }\end{array}$ & 93 & 14.50 & 30.30 & 0.879 & & & & 17.50 & 16.80 & 1.042 \\
\hline $\begin{array}{l}\text { Mikol DD, } \\
\text { et al. [32] }\end{array}$ & 22 & $\begin{array}{l}\text { Glatiramer acetate vs. } \\
\text { IFNB-1a }\end{array}$ & 585 & & & & 0.82 & 0.67 & 1.224 & 8.70 & 11.70 & 0.744 \\
\hline $\begin{array}{l}\text { Ravnborg } \\
\text { M, et al. [37] }\end{array}$ & 36 & $\begin{array}{l}\text { IFNB-1a plus } \\
\text { Methylprednisolone } \\
\text { vs. IFNB-1a }\end{array}$ & 220 & -1.06 & 1.21 & 0.978 & 5.20 & 8.00 & 0.650 & 25.00 & 28.50 & 0.877 \\
\hline $\begin{array}{l}\text { Sorensen } \\
\text { PS, et al. [39] }\end{array}$ & 22 & $\begin{array}{l}\text { IFNB-1a plus } \\
\text { Methylprednisolone } \\
\text { Vs. IFNB-1a }\end{array}$ & 110 & & & & 2.70 & 3.50 & 0.771 & 16.00 & 25.00 & 0.640 \\
\hline $\begin{array}{l}\text { O'Connor P, } \\
\text { et al. [34] }\end{array}$ & 42 & $\begin{array}{l}\text { Glatiramer acetate vs. } \\
\text { IFNB-1b } 250 \mu \mathrm{g}\end{array}$ & 913 & 17.00 & 10.00 & 1.064 & 4.60 & 3.30 & 1.394 & 20.50 & 22.30 & 0.919 \\
\hline $\begin{array}{l}\text { O'Connor P, } \\
\text { et al. [34] }\end{array}$ & 42 & $\begin{array}{l}\text { Glatiramer acetate vs. } \\
\text { IFNB-1b } 500 \mu \mathrm{g}\end{array}$ & 971 & 17.00 & 12.00 & 1.045 & 4.60 & 3.30 & 1.394 & 20.50 & 22.30 & 0.919 \\
\hline $\begin{array}{l}\text { Freedman } \\
\text { MS, et al. [21] }\end{array}$ & 36 & 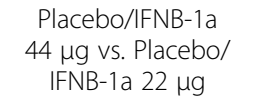 & 53 & -3.40 & 1.10 & 0.955 & 2.00 & 1.70 & 1.176 & 46.00 & 40.00 & 1.150 \\
\hline $\begin{array}{l}\text { Freedman } \\
\text { MS, et al. [21] }\end{array}$ & 36 & $\begin{array}{l}\text { Placebo/IFNB-1a } \\
44 \mu \mathrm{g} \text { vs. IFNB-1a } \\
22 \mu \mathrm{g}\end{array}$ & 83 & 5.40 & 1.10 & 1.043 & 1.70 & 1.70 & 1.000 & 39.00 & 40.00 & 0.975 \\
\hline $\begin{array}{l}\text { Freedman } \\
\text { MS, et al. [21] }\end{array}$ & 36 & $\begin{array}{c}\text { Placebo/IFNB-1a } \\
44 \mu \mathrm{g} \text { vs. IFNB-1a } \\
22 \mu \mathrm{g}\end{array}$ & 85 & 1.80 & 1.10 & 1.007 & 1.30 & 1.70 & 0.765 & 35.00 & 40.00 & 0.875 \\
\hline $\begin{array}{l}\text { Coles AJ, } \\
\text { et al. [16] }\end{array}$ & 36 & $\begin{array}{c}\text { Alemtuzumab vs. } \\
\text { IFNB-1a }\end{array}$ & 227 & -16.40 & -13.30 & 0.964 & & & & 9.00 & 26.20 & 0.344 \\
\hline
\end{tabular}


Table 2 Study summaries in trials reporting MRI changes and disability progression (Continued)

\begin{tabular}{|c|c|c|c|c|c|c|c|c|c|c|c|c|}
\hline $\begin{array}{l}\text { Kappos } L_{,} \\
\text {et al. [30] }\end{array}$ & 24 & $\begin{array}{l}\text { Fingolimod } 0.5 \mathrm{mg} \text { vs. } \\
\text { Placebo }\end{array}$ & 537 & -1.69 & 8.61 & 0.905 & 2.50 & 9.80 & 0.255 & 17.70 & 24.10 & 0.734 \\
\hline $\begin{array}{l}\text { Kappos } L, \\
\text { et al. [30] }\end{array}$ & 24 & $\begin{array}{l}\text { Fingolimod } 1.25 \text { mg } \\
\text { vs. Placebo }\end{array}$ & 512 & -3.10 & 8.61 & 0.892 & 2.50 & 9.80 & 0.255 & 16.60 & 24.10 & 0.689 \\
\hline $\begin{array}{l}\text { Polman CH, } \\
\text { et al. [36] }\end{array}$ & 24 & $\begin{array}{l}\text { Natalizumab vs. } \\
\text { Placebo }\end{array}$ & 942 & & & & 1.90 & 11.00 & 0.173 & 17.00 & 29.00 & 0.586 \\
\hline $\begin{array}{l}\text { Clanet M } \\
\text { et al. [14] }\end{array}$ & 36 & $\begin{array}{l}\text { IFNB-1a } 60 \mu \mathrm{g} \text { vs. } \\
\text { IFNB-1a } 30 \mu \mathrm{g}\end{array}$ & 386 & -0.20 & -1.29 & 1.011 & 8.00 & 9.00 & 0.889 & 37.00 & 37.00 & 1.000 \\
\hline $\begin{array}{l}\text { Ebers GC, } \\
\text { et al. [9] }\end{array}$ & & $\begin{array}{l}\text { IFNB-1a } 22 \mu \mathrm{g} \text { vs. } \\
\text { Placebo }\end{array}$ & 279 & -1.20 & 10.90 & 0.891 & 9.00 & 15.50 & 0.581 & 30.00 & 38.00 & 0.789 \\
\hline $\begin{array}{l}\text { Ebers GC, } \\
\text { et al. [9] }\end{array}$ & & $\begin{array}{l}\text { IFNB-1a } 44 \mu \mathrm{g} \text { vs. } \\
\quad \text { Placebo }\end{array}$ & 281 & -3.80 & 10.90 & 0.867 & 5.50 & 15.50 & 0.355 & 26.50 & 38.00 & 0.697 \\
\hline $\begin{array}{l}\text { Baumhackl } \\
\text { U, et al. [13] }\end{array}$ & 24 & $\begin{array}{l}\text { Hydrolytic enzymes } \\
\text { vs. Placebo }\end{array}$ & 291 & -1.00 & -1.00 & 1.000 & & & & 28.00 & 26.00 & 1.077 \\
\hline $\begin{array}{l}\text { Millefiorini } \\
\text { E, et al. [33] }\end{array}$ & 24 & $\begin{array}{l}\text { Mitoxantrone vs. } \\
\text { Placebo }\end{array}$ & 42 & & & & 3.50 & 7.30 & 0.479 & 7.00 & 37.00 & 0.189 \\
\hline $\begin{array}{l}\text { Giovannoni } \\
\text { G, et al. [22] }\end{array}$ & 22 & $\begin{array}{c}\text { Cladribine } 5.25 \mathrm{mg} / \mathrm{kg} \\
\text { bw vs. Placebo }\end{array}$ & 674 & & & & 0.33 & 1.43 & 0.231 & 15.10 & 20.60 & 0.733 \\
\hline $\begin{array}{l}\text { Giovannoni } \\
\text { G, et al. [22] }\end{array}$ & 22 & $\begin{array}{c}\text { Cladribine } 3.5 \mathrm{mg} / \mathrm{kg} \\
\text { bw vs. Placebo }\end{array}$ & 651 & & & & 0.38 & 1.43 & 0.266 & 14.30 & 20.60 & 0.694 \\
\hline $\begin{array}{l}\text { Jacobs LD, } \\
\text { et al. [26] }\end{array}$ & 24 & IFNB-1a vs. Placebo & 164 & -13.20 & -6.50 & 0.928 & & & & 21.10 & 33.30 & 0.633 \\
\hline $\begin{array}{l}\text { Ebers GC, } \\
\text { et al. [8] }\end{array}$ & 36 & $\begin{array}{c}\text { IFNB-1b } 1.6 \text { MIU vs. } \\
\text { Placebo }\end{array}$ & 164 & 0.20 & 15.00 & 0.871 & 1.80 & 4.90 & 0.367 & 28.00 & 28.00 & 1.000 \\
\hline $\begin{array}{l}\text { Ebers GC, } \\
\text { et al. [8] }\end{array}$ & 36 & $\begin{array}{l}\text { IFNB-1b } 8 \text { MIU vs. } \\
\text { Placebo }\end{array}$ & 167 & -9.30 & 15.00 & 0.789 & 2.00 & 4.90 & 0.408 & 20.00 & 28.00 & 0.714 \\
\hline $\begin{array}{l}\text { Panitch H, } \\
\text { et al. [35] }\end{array}$ & 36 & $\begin{array}{c}\text { IFNB-1b } 160 \mu \mathrm{g} \text { vs. } \\
\text { Placebo }\end{array}$ & 81 & 0.80 & 10.90 & 0.909 & & & & 39.00 & 34.00 & 1.147 \\
\hline $\begin{array}{l}\text { Panitch H, } \\
\text { et al. [35] }\end{array}$ & 36 & $\begin{array}{c}\text { IFNB-1b } 250 \mu \mathrm{g} \text { vs. } \\
\text { Placebo }\end{array}$ & 81 & 0.40 & 10.90 & 0.905 & & & & 32.00 & 34.00 & 0.941 \\
\hline $\begin{array}{l}\text { Edan G, } \\
\text { et al. [41] }\end{array}$ & 36 & $\begin{array}{c}\text { Mitoxantrone } 12 \mathrm{mg} / \\
\mathrm{m}^{2} \text { vs. IFN } \beta-1 \mathrm{~b} \\
250 \mu \mathrm{g}\end{array}$ & 109 & & & & 2.15 & 3.50 & 0.614 & 9.10 & 25.90 & 0.351 \\
\hline $\begin{array}{l}\text { Freedman } \\
\text { MS, et al. [42] }\end{array}$ & 24 & $\begin{array}{c}\text { MBP8298 vs. Placebo } \\
\text { in DR2+ and/or DR4+ } \\
\text { haplotypes }\end{array}$ & 513 & 0.90 & 6.20 & 0.95 & 3.90 & 3.30 & 1.182 & 30.70 & 27.80 & 1.104 \\
\hline $\begin{array}{l}\text { Freedman } \\
\text { MS, et al. [42] }\end{array}$ & 24 & $\begin{array}{c}\text { MBP8298 vs. Placebo } \\
\text { in DR2- and/or DR4- } \\
\text { haplotypes }\end{array}$ & 99 & 16.10 & 0.70 & 1.15 & 3.20 & 3.30 & 0.970 & 28.30 & 35.80 & 0.791 \\
\hline $\begin{array}{l}\text { Comi G, } \\
\text { et al. [45] }\end{array}$ & 24 & $\begin{array}{l}\text { Laquinimod } 0.6 \text { mg } \\
\text { vs. Placebo }\end{array}$ & 1,106 & & & & 5.03 & 7.14 & 0.704 & 11.10 & 15.70 & 0.707 \\
\hline
\end{tabular}


Table 2 Study summaries in trials reporting MRI changes and disability progression (Continued)

\begin{tabular}{|c|c|c|c|c|c|c|c|c|c|c|c|c|}
\hline $\begin{array}{l}\text { Cohen JA, } \\
\text { et al. [43] }\end{array}$ & 24 & $\begin{array}{l}\text { Alemtuzumab } 12 \mathrm{mg} \\
\text { vs. IFN } \beta-1 \mathrm{a} 44 \mathrm{\mu g}\end{array}$ & 563 & -9.30 & -6.50 & 0.97 & & & & 8.00 & 11.12 & 0.719 \\
\hline $\begin{array}{l}\text { Coles AJ, } \\
\text { et al. [44] }\end{array}$ & 24 & $\begin{array}{l}\text { Alemtuzumab } 12 \mathrm{mg} \\
\text { vs. IFN } \beta-1 \mathrm{a} 44 \mathrm{\mu g}\end{array}$ & 667 & -1.27 & -1.23 & 1.00 & & & & 12.71 & 21.13 & 0.602 \\
\hline $\begin{array}{l}\text { Gold R, } \\
\text { et al. [47] }\end{array}$ & 24 & $\begin{array}{l}\text { Dimethyl fumarate } \\
240 \text { mg BID vs. } \\
\text { Placebo }\end{array}$ & 818 & & & & 2.60 & 17.00 & 0.153 & 16.00 & 27.00 & 0.593 \\
\hline $\begin{array}{l}\text { Gold R, } \\
\text { et al. [47] }\end{array}$ & 24 & $\begin{array}{l}\text { Dimethyl fumarate } \\
240 \text { mg TID vs. } \\
\text { Placebo }\end{array}$ & 824 & & & & 4.40 & 17.00 & 0.259 & 18.00 & 27.00 & 0.667 \\
\hline $\begin{array}{l}\text { Fox RJ, } \\
\text { et al. [46] }\end{array}$ & 24 & $\begin{array}{l}\text { Dimethyl fumarate } \\
240 \text { mg BID vs. } \\
\text { Placebo }\end{array}$ & 480 & & & & 5.10 & 17.40 & 0.293 & 13.00 & 17.00 & 0.765 \\
\hline $\begin{array}{l}\text { Fox RJ, } \\
\text { et al. [46] }\end{array}$ & 24 & $\begin{array}{l}\text { Dimethyl fumarate } \\
240 \text { mg TID vs. } \\
\text { Placebo }\end{array}$ & 466 & & & & 4.70 & 17.40 & 0.270 & 13.00 & 17.00 & 0.765 \\
\hline $\begin{array}{l}\text { Fox RJ, } \\
\text { et al. [46] }\end{array}$ & 24 & $\begin{array}{c}\text { Glatiramer acetate vs. } \\
\text { Placebo }\end{array}$ & 471 & & & & 8.00 & 17.40 & 0.460 & 16.00 & 17.00 & 0.941 \\
\hline
\end{tabular}

BID, twice daily; EDSS, Expanded Disability Status Scale; IFNB, Interferon-beta; $\mu \mathrm{g}$, microgram; $\mathrm{mg}$, milligram; $\mathrm{mg} / \mathrm{m} 2$, milligrams per square meter of body surface; mg/kg bw, milligram per kilogram of body weight; MIU, million international units; TID, three times daily. 
Table 3 Results of the regression analysis

\begin{tabular}{|c|c|c|c|c|c|c|}
\hline Model & Predictor & Slope & $p$-value & Lower $95 \% \mathrm{Cl}$ & Upper $95 \% \mathrm{Cl}$ & Adjusted $\mathrm{R}^{2}$ \\
\hline RRMS, All DMTs & Annual relapse ratio log-ratio & 0.531 & $<0.001$ & 0.425 & 9.637 & 0.750 \\
\hline RRMS, Approved DMTs & Annual relapse ratio log-ratio & 0.500 & $<0.001$ & 0.338 & 0.663 & 0.670 \\
\hline SPMS and SPMS Mixed & Annual relapse ratio log-ratio & 0.397 & 0.027 & 0.060 & 0.735 & 0.414 \\
\hline RRMS, All DMTs & T2 median volume log-ratio & 1.953 & 0.015 & 0.428 & 3.477 & 0.247 \\
\hline RRMS, Approved DMTs & T2 median volume log-ratio & 1.407 & 0.018 & 0.281 & 2.533 & 0.3305 \\
\hline RRMS, Not Approved & T2 median volume log-ratio & 3.978 & 0.151 & -2.055 & 10.011 & 0.238 \\
\hline SPMS and SPMS Mixed & T2 median volume log-ratio & NA & NA & NA & NA & NA \\
\hline RRMS, All DMTs & T2 lesion count log-ratio & 0.138 & $<0.001$ & 0.079 & 0.196 & 0.706 \\
\hline RRMS, Approved DMTs & T2 lesion count log-ratio & 0.162 & 0.025 & 0.027 & 0.298 & 0.467 \\
\hline SPMS and SPMS Mixed & T2 lesion count log-ratio & NA & NA & NA & NA & NA \\
\hline
\end{tabular}

DMT, disease-modifying treatment; RRMS, relapsing-remitting multiple sclerosis; SPMS, secondary progressive multiple sclerosis; NA, not available.

lower: 0.34. This is perhaps unsurprising, given the wide confidence interval for the slope (0.43-3.48); while we see a significant relationship between median T2 percentage volume and EDSS progression, it is not one that is precisely estimated.

Given the large difference in estimated slope between RRMS, approved DMTs, and RRMS overall, sensitivity analyses were conducted to explore the difference. A separate analysis was conducted limited to studies for which the active treatment has not been approved. The relationship between T2 lesion volume difference and the EDSS relative risk was much stronger in the RRMS studies lacking an approved DMT (slope $=3.98,95 \%$ CI: $-2.06-10.01$ ) but was not statistically significant $(p=0.15)$. The high slope but wide confidence interval suggests that in these studies, T2 lesion volume changes much less, relative to change in EDSS progression, than in studies with approved DMTs. Specifically, there are sometimes small differences in lesion volume even though there are large differences in the percentage of patients with EDSS progression. This may be due to a different mechanism of action, but also may simply be an artifact of sampling error, given the wide confidence interval. If this signal is not an artifact, it may suggest that newer and/or less conventional therapies can affect lesion volume very little relative to their effect on short-term disease progression.

\section{Prediction of EDSS progression using (log)-ratio of mean number of active T2 lesions (RRMS, all DMTs, RRMS, approved DMTs)}

The slopes for the prediction of the log-relative-risk of EDSS progression from the log-ratio of T2 lesions (generally defined as "new or enlarging" T2 lesions) were statistically significant $(0.23$ and 0.21 respectively, $p<0.001)$ for both the set of all RRMS studies and the set restricted to studies comparing approved DMTs. There were insufficient data on T2 lesion counts to conduct analyses on SPMS patients.
Following methods used previously, the median ratio of follow-up T2 lesion volume was 0.48 . For instance, in Millefiorini et al. [33] the mean number of active T2 lesions was 7.3 in the placebo group and 3.5 in the mitoxantrone group $(3.5 / 7.3=0.48$, log-ratio $=-0.74)$.

The predicted difference between treatments in EDSS progression in studies like Millefiorini et al. [33] is:

$$
\ln \left(\mathrm{RR}_{\mathrm{EDSS}}\right)=0.23 *(-0.74)=-0.17
$$

As $\exp (-0.17)=0.84$, we predict that the relative risk of EDSS progression in studies like Millefiorini et al. will be 0.84 . The actual ratio in Millefiorini et al. was much lower: 0.19 ; this study was an outlier, with only $7 \%$ of patients progressing on active treatment, while $37 \%$ progressed on placebo. However, the study had a very small sample size of the study (42 patients with MRI data), making it unsurprising that the predicted value is not close to the study value. Figure 4 plots the relationship between the log-rate ratio of active T2 lesion count and log-relative risk of EDSS progression for all RRMS studies.

\section{Discussion}

This literature review and analysis demonstrates significant links between the therapeutic impact of DMTs on EDSS progression and changes on surrogate markers of disability, namely annualized relapse rates and T2 lesion counts and volumes. This analysis improves upon the Sormani et al. [6] analysis by including DMT studies of SPMS, including RRMS studies published through June of 2013, using revised data from the O'Connor et al. [34] study, examining T2 lesion volume in addition to counts of new and enlarging lesions, and exclusion of an intercept in the regression analyses. Even though our methodological approach was different, we found substantively similar results. 
While conclusions cannot be drawn regarding the impact of treating relapse on long-term disability progression, this analysis confirms that these relationships exist over the short term (approximately two years) after treatment initiation. We found a substantive clinically and statistically significant link between concurrent treatment effects in relapse rate and in EDSS. Specifically, studies with lower relative differences in relapse rate had lower relative differences in EDSS progression: the stronger the treatment effect on reducing ARR, the stronger the effect found for reducing disease progression. There was no conclusive evidence that the relationships had different strengths between approved and non-approved DMTs in RRMS patients or between types of MS (RRMS and SPMS) patients, but the sparseness of data and the presence of outliers in some analyses prohibit strong generalizations. It remains possible that differences in the mechanism of action between newer DMTs and older DMTs may result in different relapse/ EDSS relationships. However, despite our review including more than twice as many studies compared with Sormani et al. (40 vs. 19), several of which evaluated more recently approved DMTs such as dimethyl fumarate, laquinimod, and fingolimod, the findings in the two analyses are similar.

Significant association between T2 lesion measures and EDSS measures were also found. Because not all studies contributed data on all predictors and outcomes of interest (e.g., not all studies reporting median $\mathrm{T} 2$ volume change also reported the number of active $\mathrm{T} 2$ lesions, and vice versa), it is difficult to make conclusions with regard to what predictors and outcome pairs have the strongest relationships. However, the values of adjusted- $\mathrm{R}^{2}$ were higher and the relative confidence-interval widths were narrower when the log-ratio of active T2 lesion counts was the predictor of EDSS progression. It may be that the wide variation present in T2 lesion volume blurs its relationship with disability progression. Our results suggest that counting new and/or enlarged lesions is a better way to predict concurrent disability progression than lesion volume, but more research is needed to confirm this supposition.

Since this analysis used only aggregated summary data from published studies, we cannot necessarily assume that any statistical association observed between grouplevel variables may be translated to patient-level associations. Therefore, our findings cannot be used to predict any outcome at the patient-level. In addition, unlike some surrogate endpoint analyses [7], the relationships in the current investigation are concurrent. Thus, even though relationships were found between, for example, ARR ratios and relative risk of EDSS progression, it does not guarantee that early differences in relapse rate can predict later differences in EDSS progression.

\section{Conclusions}

While it remains possible that early relapses are concomitant with, rather than causative of, disease progression, treatment differences in relapse reduction are significantly related to differences in disease progression over the first two years of treatment. Similarly, treatment differences in T2 lesion measures are also predictive of treatment differences in relapse rates over the first two years.

\section{Competing interests}

Kyle Fahrbach, Rachel Huelin, and Amber Martin are employees of Evidera, which received funding from Novartis Pharmaceuticals Corporation to conduct the study on which this manuscript is based. Homa Dastani was an employee of Novartis at the time the work was completed but is now employed by BristolMyers Squibb Company. Manoj Malhotra was an employee of Novartis at the time the work was completed but is now employed by Questcor Pharmaceuticals. Edward Kim is an employee of Novartis. Stephen Rao received honoraria from Novartis for consulting on this project.

\section{Authors' contributions}

All authors were involved in developing the design for the study. KF, RH and AM carried out the design and interpretation of results. KF conducted all analyses. All authors have read and approved the manuscript.

\section{Acknowledgements}

This study contains original data. Kyle Fahrbach, PhD, has full access to the data in the study and takes responsibility for the integrity of the data and the accuracy of the data analysis. This study and preparation of the manuscript was funded by Novartis Pharmaceuticals Corporation.

\section{Author details}

${ }^{1}$ Evidera, 430 Bedford Street, Suite 300, Lexington, MA 02420, USA. ${ }^{2}$ Novartis Pharmaceuticals Corporation, One Health Plaza, USEH 135-356, East Hanover, NJ 07936-1080, USA. ${ }^{3}$ Bristol-Myers Squibb Company, Route 206 \& Province Line Road, Princeton, NJ 08543, USA. ${ }^{4}$ Cleveland Neurological Clinic, Cleveland, OH 44195, USA. ${ }^{5}$ Questcor Pharmaceuticals, 26118 Research Road, Hayward, CA 94545, USA.

Received: 3 January 2013 Accepted: 4 November 2013 Published: 19 November 2013

\section{References}

1. Goodin DS, Frohman EM, Garmany GP Jr, Halper J, Likosky WH, Lublin FD, Silberberg DH, Stuart WH, van den Noort S, et al: Disease modifying therapies in multiple sclerosis: report of the therapeutics and technology assessment subcommittee of the American Academy of Neurology and the MS council for clinical practice guidelines. Neurology 2002, 58:169-178.

2. Bramow S, Frischer JM, Lassmann H, Koch-Henriksen N, Lucchinetti CF, Sorensen PS, Laursen H: Demyelination versus remyelination in progressive multiple sclerosis. Brain 2010, 133:2983-2998.

3. Hirst C, Ingram G, Pearson O, Pickersgill T, Scolding N, Robertson N: Contribution of relapses to disability in multiple sclerosis. J Neurol 2008, 255:280-287.

4. Rio J, Rovira A, Tintore M, Huerga E, Nos C, Tellez N, Tur C, Comabella M, Montalban X: Relationship between MRI lesion activity and response to IFN-beta in relapsing-remitting multiple sclerosis patients. Mult Scler 2008, 14:479-484.

5. Weinshenker BG, Bass B, Rice GP, Noseworthy J, Carriere W, Baskerville J, Ebers GC: The natural history of multiple sclerosis: a geographically based study: I: clinical course and disability. Brain 1989, 112(Pt 1):133-146.

6. Sormani MP, Bonzano L, Roccatagliata L, Mancardi GL, Uccelli A, Bruzzi P: Surrogate endpoints for EDSS worsening in multiple sclerosis: a meta-analytic approach. Neurology 2010, 75:302-309.

7. Johnson KR, Ringland C, Stokes BJ, Anthony DM, Freemantle N, Irs A, Hill SR, Ward RL: Response rate or time to progression as predictors of survival in trials of metastatic colorectal cancer or non-small-cell lung cancer: a meta-analysis. The Lancet Oncology 2006, 7:741-746. 
8. Ebers GC: Interferon beta-1b is effective in relapsing-remitting multiple sclerosis: I: clinical results of a multicenter, randomized, double-blind, placebo-controlled trial: the IFNB multiple sclerosis study group. Neurology 1993, 43:655-661.

9. Ebers GC: Randomised double-blind placebo-controlled study of interferon beta-1a in relapsing/remitting multiple sclerosis: PRISMS (Prevention of Relapses and disability by interferon beta-1a subcutaneously in multiple sclerosis) study group. Lancet 1998, 352:1498-1504

10. Francis $\mathrm{G}$ : Randomized controlled trial of interferon- beta-1a in secondary progressive MS: Clinical results. Neurology 2001, 56:1496-1504

11. Achiron A, Gabbay U, Gilad R, Hassin-Baer S, Barak Y, Gornish M, Elizur A, Goldhammer $Y$, Sarova-Pinhas I: Intravenous immunoglobulin treatment in multiple sclerosis: effect on relapses. Neurology 1998, 50:398-402.

12. Andersen O, Elovaara I, Farkkila M, Hansen HJ, Mellgren SI, Myhr KM, Sandberg-Wollheim M, Soelberg Sorensen P: Multicentre, randomised, double blind, placebo controlled, phase III study of weekly, low dose, subcutaneous interferon beta-1a in secondary progressive multiple sclerosis. J Neurol Neurosurg Psychiatry 2004, 75:706-710.

13. Baumhackl U, Kappos L, Radue EW, Freitag P, Guseo A, Daumer M, Mertin J: A randomized, double-blind, placebo-controlled study of oral hydrolytic enzymes in relapsing multiple sclerosis. Mult Scler 2005, 11:166-168.

14. Clanet M, Radue EW, Kappos L, Hartung HP, Hohlfeld R, Sandberg-Wollheim M, Kooijmans-Coutinho MF, Tsao EC, Sandrock AW: A randomized, double-blind, dose-comparison study of weekly interferon beta-1a in relapsing MS. Neurology 2002, 59:1507-1517.

15. Cohen JA, Cutter GR, Fischer JS, Goodman AD, Heidenreich FR, Kooijmans MF, Sandrock AW, Rudick RA, Simon JH, Simonian NA, et al: Benefit of interferon (beta)-1a on MSFC progression in secondary progressive MS. Neurology 2002, 59:679-687.

16. Coles AJ, Compston DAS, Selmaj KW, Lake SL, Moran S, Margolin DH, Norris K, Tandon PK: Alemtuzumab vs. interferon beta-1a in early multiple sclerosis. N Engl J Med 2008, 359:1786-1801

17. Comi G, Filippi M, Wolinsky JS: European/Canadian multicenter, double-blind, randomized, placebo-controlled study of the effects of glatiramer acetate on magnetic resonance imaging-measured disease activity and burden in patients with relapsing multiple sclerosis. Ann Neurol 2001, 49:290-297.

18. Durelli L, Verdun E, Barbero P, Bergui M, Versino E, Ghezzi A, Montanari E, Zaffaroni M: Every-other-day interferon beta-1b versus once-weekly interferon beta-1a for multiple sclerosis: results of a 2-year prospective randomised multicentre study (INCOMIN). Lancet 2002, 359:1453-1460.

19. Etemadifar M, Janghorbani M, Shaygannejad V: Comparison of Betaferon, Avonex, and Rebif in treatment of relapsing-remitting multiple sclerosis. Acta Neurol Scand 2006, 113:283-287.

20. Fazekas F, Deisenhammer F, Strasser-Fuchs S, Nahler G, Mamoli B: Randomised placebo-controlled trial of monthly intravenous immunoglobulin therapy in relapsing-remitting multiple sclerosis: Austrian immunoglobulin in multiple sclerosis study group. Lancet 1997, 349:589-593.

21. Freedman MS, Francis GS, Sanders EA, Rice GP, O'Connor P, Comi G, Duquette P, Metz L, Murray TJ, Bouchard JP, et al: Randomized study of once-weekly interferon beta-1la therapy in relapsing multiple sclerosis: three-year data from the OWIMS study. Mult Scler 2005, 11:41-45.

22. Giovannoni G, Comi G, Cook S, Rammohan K, Rieckmann P, Sorensen PS, Vermersch P, Chang P, Hamlett A, Musch B, Greenberg SJ: A placebo-controlled trial of oral cladribine for relapsing multiple sclerosis. N Engl J Med 2010, 362:416-426.

23. Gonsette RE, Sindic C, D'Hooghe MB, De Deyn PP, Medaer R, Michotte A, Seeldrayers P, Guillaume D: Boosting endogenous neuroprotection in multiple sclerosis: the ASsociation of inosine and interferon (beta) in relapsing-remitting multiple sclerosis (ASIIMS) trial. Mult Scler 2010, 16:455-462.

24. Hartung HP, Gonsette R, Konig N, Kwiecinski H, Guseo A, Morrissey SP, Krapf H, Zwingers T, Albrecht $\mathrm{H}$, Basedow-Rajwich $\mathrm{B}$, et al: Mitoxantrone in progressive multiple sclerosis: a placebo-controlled, double-blind, randomised, multicentre trial. Lancet 2002, 360:2018-2025.

25. Havrdova E, Zivadinov R, Krasensky J, Dwyer MG, Novakova I, Dolezal O, Ticha V, Dusek L, Houzvickova E, Cox JL, et al: Randomized study of interferon beta-1a, low-dose azathioprine, and low-dose corticosteroids in multiple sclerosis. Mult Scler 2009, 15:965-976.

26. Jacobs LD, Cookfair DL, Rudick RA, Herndon RM, Richert JR, Salazar AM, Fischer JS, Goodkin DE, Granger CV, Simon JH, et al: Intramuscular interferon beta-1a for disease progression in relapsing multiple sclerosis: the Multiple Sclerosis Collaborative Research Group (MSCRG). Ann Neurol 1996, 39:285-294.

27. Johnson KP, Brooks BR, Cohen JA, Ford CC, Goldstein J, Lisak RP, Myers LW Panitch HS, Rose JW, Schiffer RB: Copolymer 1 reduces relapse rate and improves disability in relapsing-remitting multiple sclerosis: results of a phase III multicenter, double-blind placebo-controlled trial: the copolymer 1 multiple sclerosis study group. Neurology 1995, 45:1268-1276.

28. Kappos L: Placebo-controlled multicentre randomised trial of interferon beta-1b in treatment of secondary progressive multiple sclerosis. Lancet 1998, 352:1491-1497.

29. Kappos L, Polman CH, Freedman MS, Edan G, Hartung HP, Miller DH, Montalban X, Barkhof F, Bauer $L$, Jakobs $P$, et al: Treatment with interferon beta-1b delays conversion to clinically definite and McDonald MS in patients with clinically isolated syndromes. Neurology 2006, 67:1242-1249.

30. Kappos L, Radue EW, O'Connor P, Polman C, Hohlfeld R, Calabresi P, Selmaj K, Agoropoulou C, Leyk M, Zhang-Auberson L, Burtin P: A placebo-controlled trial of oral fingolimod in relapsing multiple sclerosis. N Engl J Med 2010, 362:387-401.

31. Lanzillo R, Orefice G, Quarantelli M, Rinaldi C, Prinster A, Ventrella G, Spitaleri D, Lus G, Vacca G, Carotenuto B, et al: Atorvastatin combined to interferon to verify the efficacy (ACTIVE) in relapsing-remitting active multiple sclerosis patients: a longitudinal controlled trial of combination therapy. Mult Scler 2010, 16:450-454.

32. Mikol DD, Barkhof F, Chang P, Coyle PK, Jeffery DR, Schwid SR, Stubinski B, Uitdehaag BM: Comparison of subcutaneous interferon beta-1a with glatiramer acetate in patients with relapsing multiple sclerosis (the REbif vs Glatiramer Acetate in Relapsing MS Disease [REGARD] study): a multicentre, randomised, parallel, open-label trial. Lancet Neurol 2008, 7:903-914.

33. Millefiorini E, Gasperini C, Pozzilli C, D'Andrea F, Bastianello S, Trojano M, Morino S, Morra VB, Bozzao A, Calo A, et al: Randomized placebo-controlled trial of mitoxantrone in relapsing-remitting multiple sclerosis: 24-month clinical and MRI outcome. J Neurol 1997, 244:153-159.

34. O'Connor P, Filippi M, Arnason B, Comi G, Cook S, Goodin D, Hartung HP, Jeffery D, Kappos L, Boateng F, et al: 250 (mu)g or 500 (mu)g interferon beta- $1 \mathrm{~b}$ versus $20 \mathrm{mg}$ glatiramer acetate in relapsing-remitting multiple sclerosis: a prospective, randomised, multicentre study. Lancet Neurol 2009, 8:889-897.

35. Panitch $\mathrm{H}$ : Interferon beta-1b in secondary progressive MS: results from a 3-year controlled study. Neurology 2004, 63:1788-1795.

36. Polman $\mathrm{CH}, \mathrm{O}^{\prime}$ Connor PW, Havrdova E, Hutchinson M, Kappos L, Miller DH, Phillips JT, Lublin FD, Giovannoni G, Wajgt A, et al: A randomized, placebocontrolled trial of natalizumab for relapsing multiple sclerosis. $N$ Engl J Med 2006, 354:899-910.

37. Ravnborg M, Sorensen PS, Andersson M, Celius EG, Jongen PJ, Elovaara I, Bartholome E, Constantinescu CS, Beer K, Garde E, Sperling B: Methylprednisolone in combination with interferon beta-1a for relapsing-remitting multiple sclerosis (MECOMBIN study): a multicentre, double-blind, randomised, placebo-controlled, parallel-group trial. Lancet Neurol 2010, 9:672-680.

38. Rudick RA, Stuart WH, Calabresi PA, Confavreux C, Galetta SL, Radue EW, Lublin FD, Weinstock-Guttman B, Wynn DR, Lynn F, et al: Natalizumab plus interferon beta-1a for relapsing multiple sclerosis. N Engl J Med 2006, 354:911-923.

39. Sorensen PS, Mellgren SI, Svenningsson A, Elovaara I, Frederiksen JL, Beiske AG, Myhr KM, Sogaard LV, Olsen IC, Sandberg-Wollheim M: NORdic trial of oral Methylprednisolone as add-on therapy to Interferon beta-1a for treatment of relapsing-remitting Multiple Sclerosis (NORMIMS study): a randomised, placebo-controlled trial. Lancet Neurol 2009, 8:519-529.

40. Van De Wyngaert FA, Beguin C, D'Hooghe MB, Dooms G, Lissoir F, Carton H, Sindic CJM: A double-blind clinical trial of mitoxantrone versus methylprednisolone in relapsing, secondary progressive multiple sclerosis. Acta Neurologica Belgica 2001, 101:210-216.

41. Edan G, Comi G, Le Page E, Leray E, Rocca MA, Filippi M: Mitoxantrone prior to interferon beta-1b in aggressive relapsing multiple sclerosis: a 3-year randomised trial. J Neurol Neurosurg Psychiatry 2011, 82:1344-1350.

42. Freedman MS, Bar-Or A, Oger J, Traboulsee A, Patry D, Young C, Olsson T, Li $D$, Hartung HP, Krantz M, et al: A phase III study evaluating the efficacy and safety of MBP8298 in secondary progressive MS. Neurology 2011, 77:1551-1560. 
43. Cohen JA, Coles AJ, Arnold DL, Confavreux C, Fox EJ, Hartung HP, Havrdova E, Selmaj KW, Weiner HL, Fisher E, et al: Alemtuzumab versus interferon beta 1a as first-line treatment for patients with relapsing-remitting multiple sclerosis: a randomised controlled phase 3 trial. Lancet 2012, 380:1819-1828.

44. Coles AJ, Twyman CL, Arnold DL, Cohen JA, Confavreux C, Fox EJ, Hartung HP, Havrdova E, Selmaj KW, Weiner HL, et al: Alemtuzumab for patients with relapsing multiple sclerosis after disease-modifying therapy: a randomised controlled phase 3 trial. Lancet 2012, 380:1829-1839.

45. Comi G, Jeffery D, Kappos L, Montalban X, Boyko A, Rocca MA, Filippi M, Group AS: Placebo-controlled trial of oral laquinimod for multiple sclerosis. N Engl J Med 2012, 366:1000-1009.

46. Fox RJ, Miller DH, Phillips JT, Hutchinson M, Havrdova E, Kita M, Yang M, Raghupathi K, Novas M, Sweetser MT, et al: Placebo-controlled phase 3 study of oral BG-12 or glatiramer in multiple sclerosis. N Engl J Med 2012, 367:1087-1097.

47. Gold R, Kappos L, Arnold DL, Bar-Or A, Giovannoni G, Selmaj K, Tornatore C, Sweetser MT, Yang M, Sheikh Sl, et al: Placebo-controlled phase 3 study of oral BG-12 for relapsing multiple sclerosis. N Engl J Med 2012, 367:1098-1107.

doi:10.1186/1471-2377-13-180

Cite this article as: Fahrbach et al:: Relating relapse and T2 lesion

changes to disability progression in multiple sclerosis: a systematic

literature review and regression analysis. BMC Neurology 2013 13:180.

\section{Submit your next manuscript to BioMed Central and take full advantage of:}

- Convenient online submission

- Thorough peer review

- No space constraints or color figure charges

- Immediate publication on acceptance

- Inclusion in PubMed, CAS, Scopus and Google Scholar

- Research which is freely available for redistribution 